\title{
Comunicación
}

\section{Osteopatía craneomandibular en un perro American Bully de siete meses de edad}

\author{
Craniomandibular osteopathy in a 7-month-old American Bully dog \\ Eben Salinas C. ${ }^{1,4}$, Jorge Matta S. ${ }^{2}$, Otto Zea M. ${ }^{3}$
}

\section{RESUMEN}

\begin{abstract}
Un canino macho de raza American Bully, de siete meses de edad, fue atendido por una estructura anormal en la región ventral en el lado izquierdo de la mandíbula. A la palpación se identificó agrandamiento de la rama mandibular izquierda, con dolor a la apertura de la boca. En la bioquímica sanguínea, la fosfatasa alcalina y el fósforo sérico aumentaron considerablemente. Los rayos X identificaron una marcada proliferación del periostio con invasión del tejido blando en la mandíbula. La tomografía computarizada descartó afecciones témporo-mandibulares. Se administró tratamiento con antiinflamatorios no esteroideos por vía oral, logrando que el paciente pueda ingerir alimentos sin molestias a las dos semanas del inicio del tratamiento. La evidencia clínica, de imágenes y de la histopatología fue compatible con un caso de osteopatía craneomandibular en una raza no predispuesta.
\end{abstract}

Palabras clave: osteopatía, osteoproliferativa, osteopetrosis, mandíbula, autolimitante

${ }^{1}$ Carrera de Medicina Veterinaria y Zootecnia, Universidad Cientifica del Sur, Lima, Perú

${ }^{2}$ Clínica Veterinaria "Miraflores», Lima, Perú

${ }^{3}$ Departamento de Nutrición, Facultad de Zootecnia, Universidad Nacional Agraria La Molina, Lima, Perú

${ }^{4}$ E-mail: esalinasc@cientifica.edu.pe; https://orcid.org/0000-0002-0435-7299

Recibido: 13 de octubre de 2020

Aceptado para publicación: 16 de marzo de 2021

Publicado: 23 de junio de 2021

CLos autores. Este artículo es publicado por la Rev Inv Vet Perú de la Facultad de Medicina Veterinaria, Universidad Nacional Mayor de San Marcos. Este es un artículo de acceso abierto, distribuido bajo los términos de la licencia Creative Commons Atribución 4.0 Internacional (CC BY 4.0) [https:// creativecommons.org/licenses/by/4.0/deed.es] que permite el uso, distribución y reproducción en cualquier medio, siempre que la obra original sea debidamente citada de su fuente original 
An 8-month-old male American Bully dog was brought to the clinic for an abnormal structure in the ventral region on the left side of the jaw. On palpation, enlargement of the left mandibular ramus was identified, with pain when opening the mouth. In blood biochemistry, alkaline phosphatase and serum phosphorus increased considerably. Xrays identified a marked proliferation of the periosteum with soft tissue invasion in the mandible. Computed tomography ruled out temporomandibular disorders. Treatment with non-steroidal anti-inflammatory drugs was administered orally, allowing the patient to eat without discomfort two weeks after the start of treatment. The clinical, imaging and histopathology evidence was compatible with a case of craniomandibular osteopathy in a non-predisposed breed.

Key words: osteopathy, osteoproliferative, osteopetrosis, mandible, self-limiting

\section{INTRODUCCIÓN}

La osteopatía craneomandibular (CMO) es un trastorno poco frecuente caracterizado por un proceso proliferativo periosteal no neoplásico en los huesos del cráneo. Los huesos afectados generalmente son las mandíbulas, las bullas timpánicas y el hueso occipital, pero también se ha reportado en los huesos frontales y parietales del cráneo (Watson et al., 1995; LaFond et al., 2002). La CMO afecta a múltiples razas; sin embargo, ocurre con mayor frecuencia en West Highland White y Scottish terriers (LaFond et al., 2002; Macedo et al., 2015; Beever et al., 2018). La enfermedad se presenta entre los 3 y 9 meses de edad, manifestando una inflamación dolorosa bilateral de las mandíbulas acompañado de letargia e inapetencia (Huchkowsky, 2002; Thompson et al., 2011; Macedo et al., 2015).

La patogenia de la OCM es desconocida, aunque se ha demostrado una herencia autosómica recesiva en perros de la raza West Highland White Terrier, así como en el humano con su equivalente clínico «enfermedad de Caffey» (Padgett y Mostosky, 1986; Hytönen et al., 2016; Vagt y Distl, 2018). También se ha postulado un elemento infeccioso o inflamatorio (Munjar et al., 1998; Trowald-Wigh et al., 2000).
El diagnóstico se sustenta en la evaluación física, así como en los hallazgos radiográficos, tomográficos o por resonancia magnética. Las radiografías tienen un valor limitado en el diagnóstico y muestran características hiperostóticas con agrandamiento simétrico en el cráneo. Estas lesiones se observan con mayor frecuencia en las mandíbulas y en las ampollas timpánicas (Huchkowsky, 2002; Thompson et al., 2011). Las tomografías y resonancia magnética presentan un mejor apoyo al diagnóstico, debido a su capacidad de evitar superposición de tejidos y su gran capacidad de evaluar el tejido blando circundante a las lesiones (Hudson et al., 1994; Matiasovic et al., 2016).

El diagnóstico definitivo de la OCM es mediante el estudio histopatológico, poniéndose de manifiesto la reabsorción osteoclástica del hueso laminar. De manera simultánea, se evidencia formación intermitente de hueso trabecular. Estos cambios dan una imagen en mosaico, en la que el hueso nuevo aparece separado del hueso maduro por unas líneas de compactación, alternancia de tejidos que resulta finalmente en el engrosamiento de las regiones óseas afectadas (Thompson et al., 2011; Pettitt et al., 2012; Beever et al., 2018). 


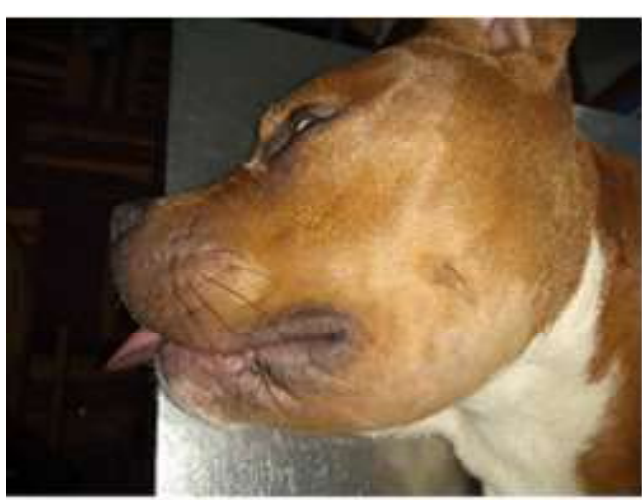

(A)

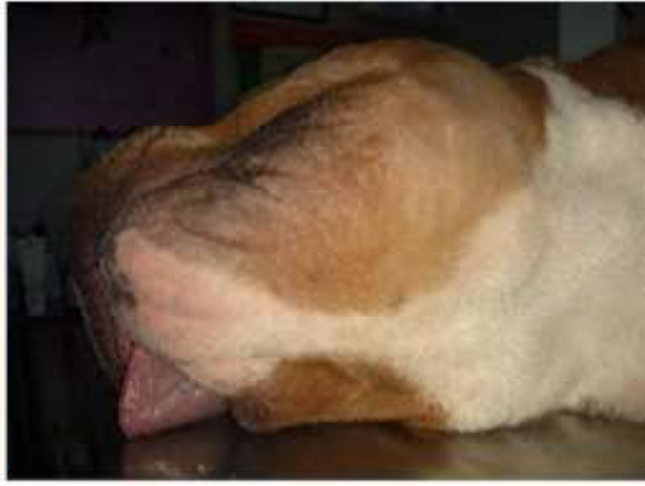

(B)

Figura 1. (A) Imagen lateral y (B) ventral de cabeza y mandíbula izquierda. La región mandibular caudal se presenta hinchada y deformada hacia ventral

\section{Caso Clínico}

\section{Anamnesis}

Se presentó para la evaluación clínica un paciente canino de raza American Bully, macho de siete meses de edad y $20 \mathrm{~kg}$ de peso. Los propietarios indicaron que desde hace dos semanas observaron una tumefacción en la mandíbula izquierda, además de dificultad para ingerir alimentos y dolor al aperturar la boca.

\section{Hallazgos clínicos}

En la evaluación física, el paciente estaba apático y letárgico, pero con una buena condición corporal. A la palpación de la región mandibular se identificó agrandamiento notorio de la rama mandibular izquierda. Los músculos faciales estaban tensos y los nódulos linfáticos submandibulares agrandados. Al aperturar la boca, el paciente demostró dolor, además de sialorrea. La temperatura corporal del perro era normal y no había signos de enfermedad sistémica (Figura 1).

\section{Ayudas Diagnósticas}

Se tomaron muestras de sangre para estudios de hemograma, orina y bioquímica completa. La fosfatasa alcalina y el fósforo sérico se encontraron elevados, mientras que la glucosa y las globulinas mostraron una ligera elevación (Cuadro 1).

Se tomaron radiografías con un equipo Perlong PLX 101C. En las radiografías de la mandíbula se observó una marcada proliferación del periostio con mayor relevancia en el lado izquierdo. El nuevo tejido óseo generó una marcada invasión del tejido blando sin márgenes definidos entre la corteza y la medula ósea.

Debido a la poca información obtenida por las radiografías, se realizó un estudio de tomografía helicoidal multicorte con un equipo Siemens Somatom Definition AS. El estudio se realizó sin administración de contraste endovenoso, con cortes axiales de $1 \mathrm{~mm}$ y con reformaciones axiales y coronales. Se identificó incremento del volumen del borde inferior del tercio medio de las ramas hori- 
Cuadro 1. Valores de hemograma en un American Bully de 7 meses de edad

\begin{tabular}{ccc}
\hline Parámetros morfológicos & Valor detectado & Valores de referencia \\
\hline Eritrocitos $\left(10^{6} / \mathrm{mm}^{3}\right)$ & 7500,000 & $5.5-8.5$ \\
Hemoglobina $(\mathrm{g} / \mathrm{dl})$ & 14.40 & $12-18$ \\
Hematocrito $(\%)$ & 43 & $37-55$ \\
Leucocitos $\left(\mathrm{x} 10^{3} / \mathrm{mm}^{3}\right)$ & 12700 & $8-16.5$ \\
Abastonados $\left(\mathrm{x} 10^{3} / \mathrm{mm}^{3}\right)$ & 0 & 0 \\
Segmentados $\left(\mathrm{x} 10^{3} / \mathrm{mm}^{3}\right)$ & 10.7 & $3-12$ \\
Eosinófilos $\left(\mathrm{x} 10^{3} / \mathrm{mm}^{3}\right)$ & 0 & $0.1-1$ \\
Basófilos $\left(\mathrm{x} 10^{3} / \mathrm{mm}^{3}\right)$ & 0 & 0 \\
Monocitos $\left(\mathrm{x} 10^{3} / \mathrm{mm}^{3}\right)$ & 1 & $0.1-1.4$ \\
Linfocitos $\left(\mathrm{x} 10^{3} / \mathrm{mm}^{3}\right)$ & 4 & $1-5$ \\
Plaquetas $\left(\mathrm{x} 10^{3} / \mathrm{mm}^{3}\right)$ & 310,000 & $200-500$ \\
\hline Parámetros bioquímicos & & $20-50$ \\
AST (UI/L) & 45 & $19-59$ \\
ALT (UI/L) & 51 & $20-279$ \\
FA (UI/L) & 363 & $5.4-7.7$ \\
Proteínas totales $(\mathrm{g} / \mathrm{dl})$ & 6.6 & $65-118$ \\
Glucosa (mg/dl) & 120 & $2.7-4.4$ \\
Globulinas $(\mathrm{g} / \mathrm{dl})$ & 4.6 & $9-12$ \\
Calcio (mg/dl) & 15 & $4-8$ \\
Fósforo (mg/dl) & 9.7 & \\
\hline AST: aspartato aminotransferasa & $\mathrm{ALT}$ & \\
\hline
\end{tabular}

AST: aspartato aminotransferasa; ALT: alanina aminotransferasa; FA: fosfatasa alcalina

zontales de la mandíbula a predominio izquierdo y dependiente de la corteza y periostio. No se observaron lesiones líticas ni blásticas definidas que sugieran procesos neoformativos (Figuras 2 y 3 ).

Posteriormente, se anestesió al paciente para realizar una toma de muestra para biopsia. Se pre medicó con Medetomidina $0.005 \mathrm{mg} / \mathrm{kg}$ más Butorfanol $0.3 \mathrm{mg} / \mathrm{kg}$, ambos por vía intramuscular. La inducción anestésica se realizó con Propofol a dosis 3 $\mathrm{mg} / \mathrm{kg}$ por vía endovenosa y el mantenimiento se realizó con Sevoflurano con una CAM de 2.8. Se tomaron biopsias de los huesos mandibulares con una aguja Jamshidi. Las muestras se extrajeron cuidadosamente e incluyeron hueso trabecular y compacto. Se lavaron con solución fisiológica y se fijaron en formalina neutra al $10 \%$, para luego preparar las láminas histológicas, que se tiñeron con hematoxilina/eosina. En la evaluación histopatológica se identificaron trabéculas gruesas, las cuales comprendían una mezcla compleja de tejido laminar inmaduro y hueso laminar maduro. Estas láminas estaban separadas por muchas líneas de cementación, algunas con bordes ondulados indicando reabsorción anterior. En algunas áreas se evidenció reabsorción activa por osteoclastos 

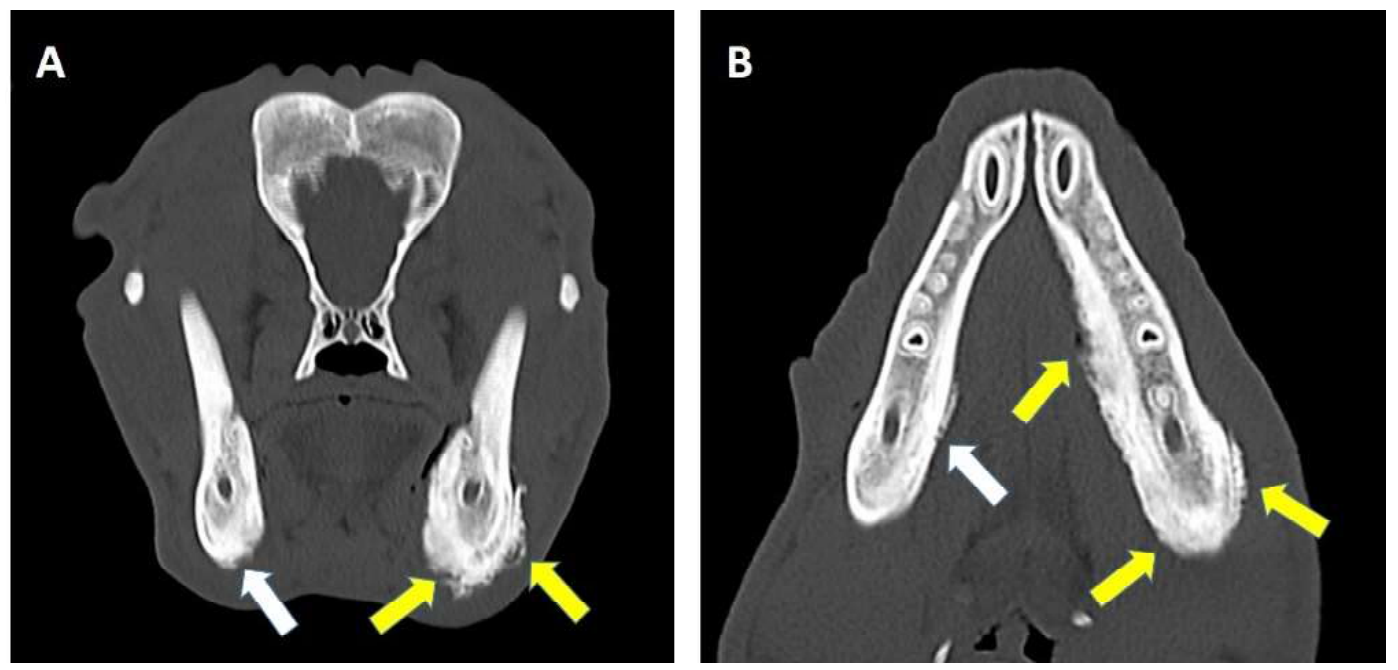

Figura 2. Tomografía computarizada en plano transverso (A) y plano dorsal (B) del cráneo y región mandibular. La nueva proliferación ósea está presente solo en la región caudal ventral y medial de la rama derecha de la mandíbula (flechas blancas) y a lo largo de las superficies ventral, medial y lateral de rama izquierda de la mandíbula (flechas amarillas)

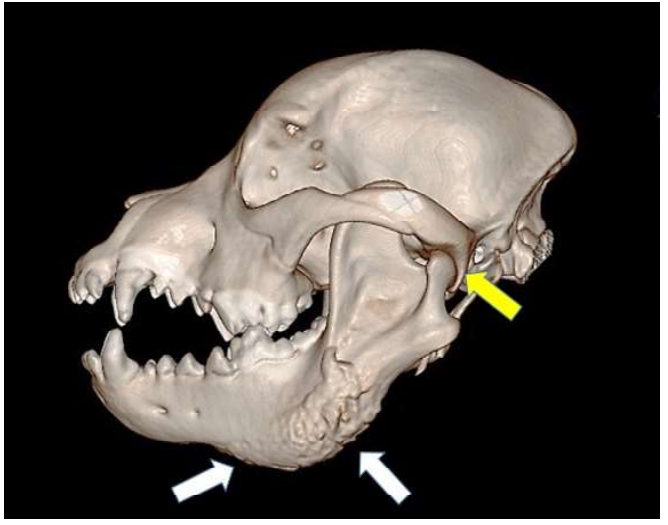

Figura 3. Reconstrucción tridimensional de la tomografía computarizada del cráneo de un perro American Bully de 7 meses con osteopatía craneomandibular. Se muestran las proliferaciones de hueso nuevo en las regiones lateral y ventral de la mandíbula izquierda (flechas blancas). No hay afectación de los senos paranasales, hueso temporal petroso del cráneo, ni de la articulación témporo mandibular (flecha amarilla) mientras que en otras regiones prominentes osteoblastos revestían las trabéculas indicando osteoproducción.

\section{Tratamiento}

Se instauró el tratamiento con el objetivo de controlar el dolor y proporcionar confort al comer. Se administró meloxicam 0.2 $\mathrm{mg} / \mathrm{kg}$ por vía oral para el primer día y luego $0.1 \mathrm{mg} / \mathrm{kg}$ por vía oral cada 24 horas durante 10 días. El dolor se redujo considerablemente, logrando el paciente ingerir alimentos sin molestias a las dos semanas de inicio del tratamiento. El tratamiento con meloxican se mantuvo de manera intermitente a cada 48 horas desde el día 11 semana hasta completar las 30 días. A los tres meses del diagnóstico y coincidiendo con la madurez esquelética, el paciente continuó libre de dolor en las regiones afectadas. 


\section{Discusión}

El paciente presentó los signos y características comunes de la CMO, como la proliferación ósea periosteal mandibular y manifestaciones clínicas como el dolor al abrir la boca. La CMO se presenta en perros en etapa de crecimiento, principalmente en la etapa prepúber (entre el tercer y octavo mes de edad) (Shorentein et al., 2014; Matiasovic et al., 2016) y afecta principalmente a los huesos de la cabeza con osificación de origen endocondral, como el occipital, las bullas timpánicas y las ramas mandibulares (Hudson et al., 1994; Taylor et al., 1995). La predisposición racial es bastante frecuente para los West Highland White Terrier (Macedo et al., 2015) y Scottish Terrier (Shorentein et al., 2014). Los autores no encontraron reportes de $\mathrm{CMO}$ en perros American Bully, por lo que podría considerarse este caso como el primer reporte para esta raza.

El paciente solo presentó lesiones óseas en la mandíbula, sin afectación de otras partes del cráneo ni huesos largos. Esto coincide con lo reportado en la distribución en las lesiones y su relación con perros no terrier. De esta forma, los perros no terrier presentan con más frecuencia lesiones solo en las ramas mandibulares (Franch et al., 1998; Huchkowsky, 2002). Los perros de raza Terrier suelen tener lesiones más invasivas y que pueden afectar, además, a las articulaciones témporo mandibulares y cráneo (Beever et al., 2018).

En relación al hemograma y bioquímica sanguínea, el paciente presentó considerable elevación de la fosfatasa alcalina y fósforo sérico, así como una ligera elevación de las globulinas. En la CMO se han reportado elevación de los valores de fósforo y calcio debido al incremento del metabolismo óseo que sufre el paciente (Watson et al., 1995; Ratterree et al., 2011). La elevación de fosfatasa alcalina ha sido asociada también en pacientes con CMO debido al aumento de la actividad osteoblástica (Huchkowsky, 2002; Ratterree et al., 2011).
Las evaluaciones por imágenes son eficientes métodos en pacientes con CMO para apoyar el diagnóstico, evaluar la evolución y brindar un pronóstico de la enfermedad. En el estudio radiográfico de este paciente solo se evidenciaron signos radiográficos evidentes en la región longitudinal de la rama izquierda de la mandíbula e incremento de tejido blando del lado derecho. En el estudio por tomografía computarizada se pudo demostrar con mayor precisión la afectación de ambas ramas de la mandíbula y, así mismo, descartar la proyección de la lesión a la articulación témporo mandibular y cráneo.

En el presente caso, el estudio de los tejidos colectados por biopsia también contribuyó a confirmar el diagnóstico de OCM. Sin embargo, las evidencias por imágenes y su relación con el estado clínico y epidemiológico presentan en la mayoría de los casos suficiente evidencia para brindar un diagnóstico definitivo (Shorentein et al., 2014; Matiasovic et al., 2016). Los patrones histopatológicos corresponden a la reunión de abundantes trabéculas óseas con engrosamiento generalizado, presentando además abundantes líneas de cementación ósea (Craig et al., 2016). La causa más aceptada para la osteoproducción en la porción ventral mandibular es una falla en el proceso natural de resorción ósea. De esta forma se distingue una marcada reducción de la osteolisis osteocítica, fenómeno que también ocasiona la muerte de numerosos osteocitos desencadenando microfracturas y el proceso inflamatorio adjunto (Alexander, 1983; Craig et al., 2016).

El tratamiento se brindó con el fin de reducir los signos clínicos que sufría el paciente y de esta manera lograr que pueda alimentarse de manera adecuada. La administración prolongada de meloxican contribuye a mejorar la condición clínica del paciente. El uso de los AINES se reporta como buena opción terapéutica para los casos de OCM (Huchkowsky, 2002; Varalho et al., 2012). En los casos que se presente un mayor dolor, generalmente por invasión de la articulación témporo mandibular, se requiere la asocia- 
ción multimodal con analgésicos opioides (tramadol $4 \mathrm{mg} / \mathrm{kg}$ ). Adicionalmente, en caso los pacientes no puedan alimentarse adecuadamente, se debe proporcionar fluidos intravenosos y soporte nutricional por sonda nasogástrica o esofágica.

La OCM no es una enfermedad que ocasione alta mortalidad; sin embargo, los propietarios usualmente deciden por la eutanasia del paciente debido al dolor severo y a la desnutrición por la incapacidad de alimentarse (Macedo et al., 2015). El pronóstico será reservado cuando las proliferaciones óseas producen anquilosis en las articulaciones témporo mandibulares o se presenten déficits neurológicos por compresión mecánica del cerebelo o tronco encefálico (Ratterree et al., 2011; Krastev et al., 2015).

\section{Conclusiones}

Se reporta un caso de osteopatía craneomandibular en un perro de 7 meses de raza no predispuesta como el American Bully. La presentación fue leve a moderada, con lesiones óseas en la mandíbula, sin afectación de otras partes del cráneo ni en huesos largos. El diagnóstico se logró sobre la base del estudio clínico, radiográfico e histopatológico. Se descartaron afecciones témporo mandibulares y craneales mediante una tomografía computarizada.

\section{Literatura Citada}

1. Alexander JW. 1983. Selected skeletal dysplasias: craniomandibular osteopathy, multiple cartilaginous exostoses, and hypertrophic osteodystrophy. Vet Clin North Am Small Anim Pract 13: 55-70. doi: 10.1016/s0195-5616(83)50004-1

2. Beever L, Swinbourne F, Priestnall S, Ter Haar G, Brockman D. 2018. Surgical management of chronic otitis secondary to craniomandibular osteopathy in three West Highland white terriers. J Small Anim Pract 60: 254-260. doi: 10.1111/jsap.12839

3. Franch J, Cesari JR, Font J. 1998. Craniomandibular osteopathy in two Pyrenean mountain dogs. Vet Rec 142: 455-459. doi: 10.1136/vr.142.17.455

4. Huchkowsky SL. 2002. Craniomandibular osteopathy in a bullmastiff. Can Vet J 43: 883-885.

5. Hudson JA, Montgomery RD, Hathcock JT, Jarboe JM. 1994. Computed tomography of craniomandibular osteopathy in a dog. Vet Radiol Ultrasound 35: 94-99. doi: 10.1111/j.17408261.1994.tb00194.x

6. Hytönen MK, Arumilli $M$, Lappalainen AK, Owczarek-Lipska M, Jagannathan V, Hundi S, Salmela $E$, et al. 2016. Molecular characterization of three canine models of human rare bone diseases: Caffey, van den Ende-Gupta, and Raine syndromes. PLoS Genet 12: e1006037. doi: 10.1371/ journal.pgen.1006037

7. Krastev SR, Simeonov $R$, Goranov $N$. 2015. Craniomandibular osteopathy in a Bulgarian shepherd dog. A case report. Trakia J Sci 13 (Suppl 2): 292-295. doi: 10.15547/tjs.2015.s.02.063

8. LaFond E, Breur GJ, Austin CC. 2002. Breed susceptibility for developmental orthopedic diseases in dogs. J Am Anim Hosp Assoc 38: 467-477. doi: $10.5326 / 0380467$

9. Macedo AS, Casagrande AJ, Friesen $R$, Minto BW. 2015. Craniomandibular osteopathy in a West Highland White Terrier. Acta Sci Vet 43: 1- 4.

10. Matiasovic M, Caine A, Scarpante E, Cherubini GB. 2016. Imaging diagnosis - magnetic resonance imaging features of craniomandibular osteopathy in an Airedale Terrier. Vet Radiol Ultrasound 57:27-29. doi: 10.1111/vru.12304

11. Munjar TA, Austin CA, Breur GJ. 1998. Comparison of risk factors for hypertrophic osteodystrophy, craniomandibular osteopathy and canine distemper virus infection. Vet Comp Orthop Traumatol 11:37-43. 
12. Padgett GA, Mostosky UV. 1986. Animal model - The mode of inheritance of craniomandibular osteopathy in West Highland White Terrier Dogs. Am J Med Genet 25: 9-13.

13. Pettitt R, Fox R, Comerford E, Newitt A. 2012. Bilateral angular carpal deformity in a dog with craniomandibular osteopathy. Vet Comp Orthop Traumatol 25: 149-54. doi: 10.3415/VCOT-11-02-0022

14. Ratterree WO, Glassman MM, Driskell EA, Havig ME. 2011. Craniomandibular osteopathy with a unique neurological manifestation in a young Akita. J Am Anim Hosp Assoc 47: e7 e12. doi: 10.5326/JAAHA-MS-5464

15. Craig LE, Dittmer KE, Thompson KG. 2016. Bones and joints. In: Maxie MG (ed). Jubb, Kennedy, and Palmer's pathology of domestic animals. $6^{\text {th }}$ ed. St. Louis: Elsevier. p 16-163.

16. Shorentein B, Schwartz P, Kross PH. 2014. Craniomandibular osteopathy in dogs. Vet Med Today What Is Your Diagnosis 245(5): 41-42. doi: 10.2460/ javma.245.5.491

17. Taylor SM, Remedios A, Myers S. 1995. Craniomandibular osteopathy in a Shetland Sheepdog. Can Vet 136: 437-439.
18. Thompson DJ, Rogers W, Owen MC, Thompson KG. 2011. Idiopathic canine juvenile cranial hyperostosis in a Pit Bull Terrier. N Z Vet J 59: 201-205. doi: 10.1080/00480169.2011.579556

19. Trowald Wigh G, Ekman S, Hansson K, Hedhammar A, Segerstad CHA. 2000. Clinical, radiological and pathological features of 12 Irish Setters with canine leucocyte adhesion deficiency. J Small Anim Pract 41: 211217. doi:10.1111/j.1748-5827.2000.tb03198.x

20. Vagt J, Distl O. 2018. Complex segregation analysis of craniomandibular osteopathy in Deutsch Drahthaar dogs. Vet J 231: 30-32. doi: 10.1016/j.tvj1.2017.11.008

21. Varalho GR, Lima BR, Raposo TMM, Daleck CR. 2012. Osteopatia crânio mandibular em Bulldog Inglês. Relato de caso. Ars Veterinaria 28: 218-221.

22. Watson ADJ, Adams WM, Thomas CB. 1995. Craniomandibular osteopathy in dogs. Comp Contin Educ Vet Pract 17: 911-922. 